\title{
Improved Handset Antenna Performance via an Electrically Extended Ground Plane
}

\author{
Shirook M. Ali, Huanhuan Gu, Kelce Wilson, and James Warden \\ Advanced Technology, Research In Motion Limited, 560 Westmount Rd. N., Waterloo, ON, Canada N2L 0A9 \\ Correspondence should be addressed to Huanhuan Gu, hgu@rim.com \\ Received 12 December 2011; Accepted 18 January 2012 \\ Academic Editors: M. Y. W. Chia and J. Park
}

Copyright () 2012 Shirook M. Ali et al. This is an open access article distributed under the Creative Commons Attribution License, which permits unrestricted use, distribution, and reproduction in any medium, provided the original work is properly cited.

A novel and practical approach is presented providing improved antenna performance without enlarging the antenna or the ground plane. The approach electrically extends the ground plane using wire(s) that behave as surface metal extensions of the ground plane. The wire extensions can be accommodated within typical handset housing or as part of the stylish metal used on the handset's exterior perimeter; hence don't require enlargement of the device. Consequently, this approach avoids the costs and limitations traditionally associated with physically lengthening of a ground plane. Eight variations are presented and compared with baseline antenna performance. Both far-field patterns and near-field electromagnetic scans demonstrate that the proposed approach controls the electrical length of the ground plane and hence its chassis wavemodes, without negatively impacting the characteristics of the antenna. Improvements in performance of up to $56 \%$ in bandwidth at $900 \mathrm{MHz}$ and up to $12 \%$ in efficiency with a reduction of up to $12 \%$ in the specific absorption rate (SAR) are achieved. An $8 \%$ increase in efficiency with a $1.3 \%$ improvement in bandwidth and a $20 \%$ reduction in SAR is achieved at $1880 \mathrm{MHz}$. Thus, improvements in bandwidth are achieved without compromising efficiency. Further, improvements at lower frequencies do not compromise performance at higher frequencies.

\section{Introduction}

Handset users increasingly demand smaller and lighter devices, coupled with long battery life. Unfortunately, compactness, which results in slim and shortened device form factors, is often achieved at the cost of degraded antenna performance. This undesirable tradeoff has inspired numerous research efforts to find a more attractive balance between providing conveniently small and stylish handset dimensions while preserving acceptable antenna performance. In this effort, the influence of the mobile-phone-environmentrelated parameters, such as chassis (ground plane) size and the antenna position on the ground plane, have been thoroughly investigated, see for example [1-8].

It is known that the total radiation bandwidth of the mobile device is defined by both antenna properties and the chassis physical dimensions [1]. In [5], the authors show that if the chassis is resonant at the antenna-operating frequency, the bandwidth will increase significantly. A more detailed analysis in [1] demonstrated the chassis effects on both specific absorption rate (SAR) and radiation efficiency. It was demonstrated that the resonance of the chassis leads to an increase in SAR and a decrease in radiation efficiency. These chassis-based analysis efforts have revealed a general trend of exploiting chassis properties for antenna design.

Previous efforts $[5,9,10]$ have consistently demonstrated that the optimal dimensions of the ground plane, for achieving the best antenna performance, are those that enable resonance. This has significant consequences for low frequencies, including the $800 \mathrm{MHz}$ and $900 \mathrm{MHZ}$ GSM bands and the proposed $700 \mathrm{MHz}$ band for LTE in the USA. However, a handset that is sized large enough to contain a ground plane that resonates at these low frequencies is often undesirable to consumers.

Some techniques have been proposed to address this issue, for example by controlling the chassis wavemodes [ 5 , $11,12]$. These showed that the incorporation of slots in the chassis plane could lead to significant bandwidth broadening without changing the chassis dimensions. However, the introduction of the quarter wavelength slot effectively acts as an introduction of another antenna, that is, a slot antenna, which resonates around the desired frequency. Therefore, 
it is the resonance of both the main antenna and this slot antenna that produces the broader bandwidth. References $[5,11]$ proposed the slot design and optimization process for a single-band broadening, centered at the lower frequency of $900 \mathrm{MHz}$, whereas [12] demonstrated that the same concept can also be applied to higher frequency bands. However, as indicated in [5], introducing these relatively large quarter wavelength slots is not realizable from a practical stand point, in addition to problematic electromagnetic (EM) interference that results from the slot's proximity to the $\mathrm{RF}$ and signal processing modules. Although the approach indicated in [11] overcomes the electromagnetic interference issues in [5] and [12], the layout of PCB design becomes complex. Thus, there have not been any techniques in the literature that control the chassis such that to electrically increase it without altering the antenna ground plane itself.

In this paper, we propose a novel approach of using a wire to electrically extend portions of the chassis ground plane without altering the chassis itself. Forming a closed loop with this wire approximates, at RF, an equivalent solid surface, have the dimensions of the closed wire loop. The dimensions of the wire loop depend on the frequency in which an improvement in the antenna performance is most needed. The wire can be used in a straight form or bent as notches in the interest of shortening its physical length or height.

The remainder of the paper is organized as follows: the proposed approach is implemented using a hex-band antenna, where the antenna is introduced in Section 2. The wired approach is introduced in Section 3. There, eight variations of the proposed approach are introduced. The results and the related discussion are presented in Section 4. The paper is concluded in Section 5.

\section{Antenna under Test}

The simulation model and prototype of the antenna under test (AUT) are illustrated in Figure 1. The antenna is a $3 \mathrm{D}$ monopole-based hex-band antenna, which it supports GSM $800 / 900 / 1800 / 1900 \mathrm{MHz}$, UMTS $2100 \mathrm{MHz}$, and Bluetooth $2400 \mathrm{MHz}$ bands [13]. It is designed on a chassis of $100 \mathrm{~mm}$ $\times 60 \mathrm{~mm} \times 1.5 \mathrm{~mm}$, which corresponds to the PCB size of a typical handset device. It is worth mentioning here that the physical dimensions of the ground plane and the antenna structures are kept unchanged throughout this investigation.

A finite difference time domain (FDTD-) based software SEMCAD [14] is used in the simulations. The simulated and measured return losses are compared in Figure 2. As shown, the correspondence between both the simulation and measurement results is quite good.

The antenna and the ground plane characteristics are discussed later in the paper through the near-field properties with the surface current distributions and the EM field scans on the hearing aid compatibility (HAC-) defined plane [15]. The characteristics are also discussed in relation to the far-field performance through the radiation pattern of the antenna. Both the near-field and the far-field discussions are carried out for the proposed models presented and are

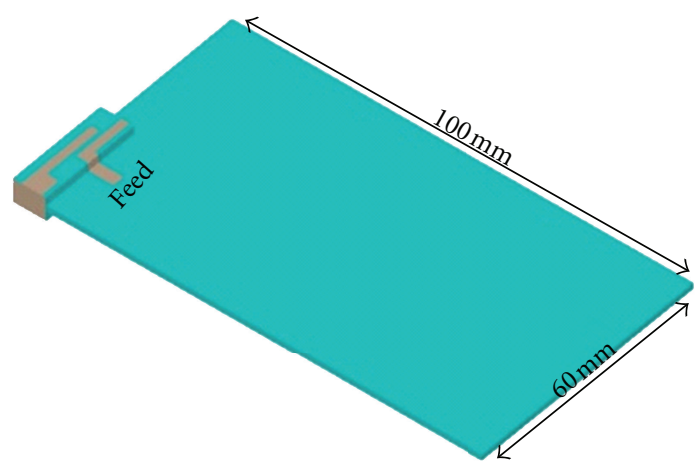

(a)

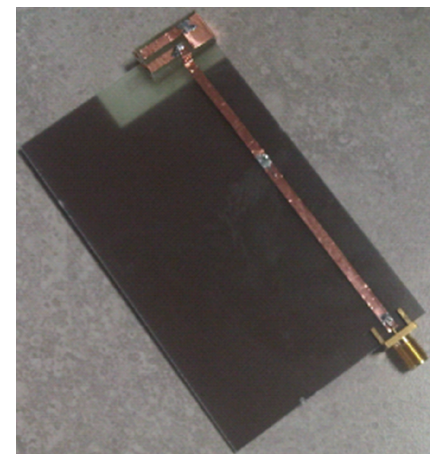

(b)

Figure 1: Antenna under test: (a) simulation model and (b) prototype.

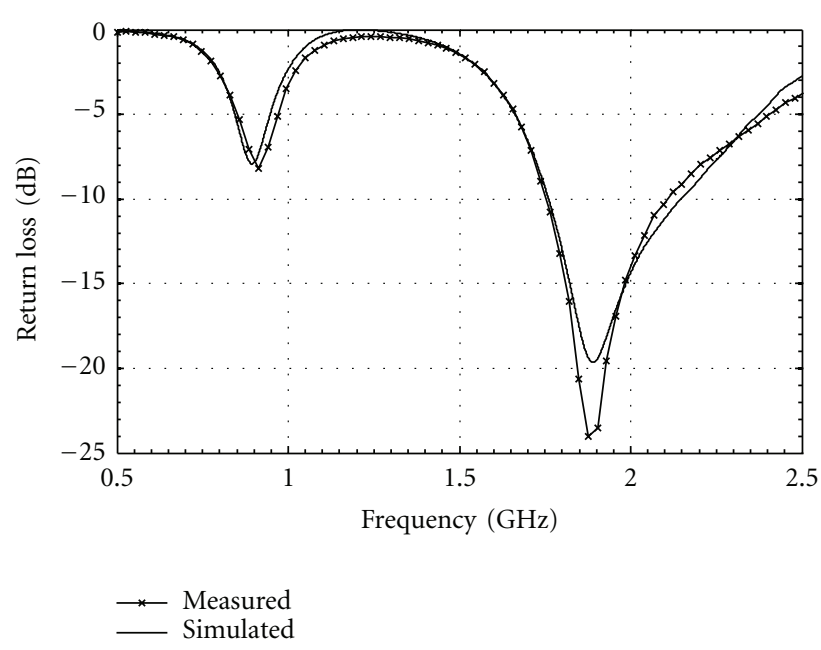

FIgURE 2: Return loss of the AUT.

compared to the initial AUT. We present in this section the near-field and the far-field characteristics of the initial AUT for comparison later in the paper.

Figure 3 illustrates the surface current distributions on the antenna and the ground plane at the frequencies of interest, $900 \mathrm{MHz}$ and $1880 \mathrm{MHz}$. As expected, the currents are concentrated on the longitudinal edges of the ground plane. Figure 4 shows the HAC scan planes for both the electrical and the magnetic field intensities at the two 


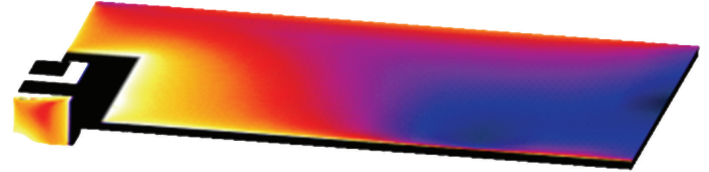

(a)

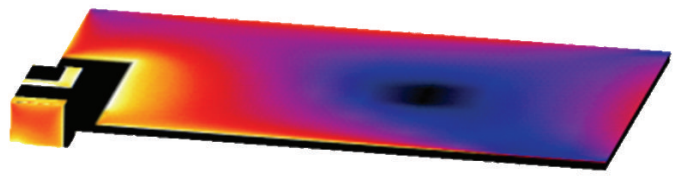

(b)

FIgURE 3: Current distribution of AUT: (a) at $900 \mathrm{MHz}$ and (b) at $1880 \mathrm{MHz}$.

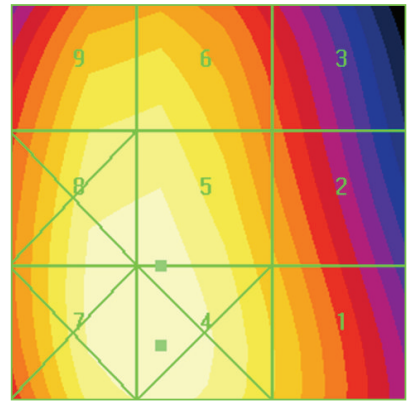

(a)

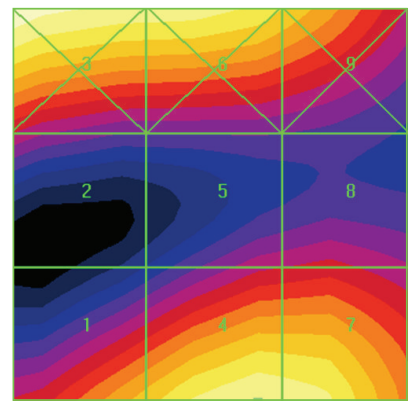

(c)

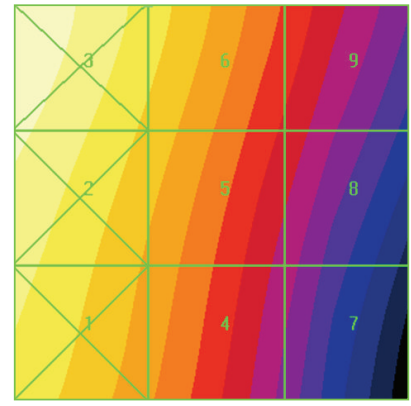

(b)

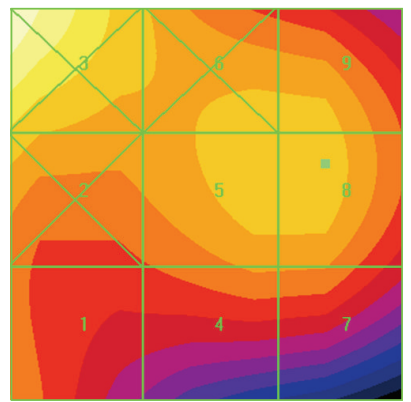

(d)
FIgURE 4: HAC-scanned result of AUT: (a) $E$ field at $900 \mathrm{MHz}$, (b) $H$ field at $900 \mathrm{MHz}$, (c) $E$ field at $1880 \mathrm{MHz}$, and (d) $H$ field at $1880 \mathrm{MHz}$.

frequencies of interest. It is worth noting here that these HAC simulations were validated, with a very good match, using extensive measurements in a HAC certified lab. For brevity, these results are not shown.

\section{Electrically Extended Chassis}

The genesis of cellular antennas lies in the field of wire antennas such as the monopole and modified monopole antennas. To improve radiation performance, these evolved into the inverted $\mathrm{F}$ antenna and the planar inverted $\mathrm{F}$ antenna that are widely used in today handsets, [16-18]. In fact, in [19], the authors proposed using the thin wire model to represent the backbone of an antenna to be designed. The antenna's conductive portions are replaced with a thin wire model, which is physically possible due to the skin effect. Indeed, if we take a closer look at the current distribution on the ground plane of the AUT in Figure 3, we notice that the current is concentrated on the ground plane edges. From this observation, we can replace the ground plane with a closed wire loop with the diameter on the surface ground plane. Or, equivalently, we can have parts of the ground plane represented as a solid metal surface, using the wires to extend it in a desired dimension.

Looking at the HAC plane field scans shown in Figure 5 for the case when the chassis physical length is increased to $120 \mathrm{~mm}$ instead of $100 \mathrm{~mm}$, we notice that the field distribution is the same. According to [10], the chassis length of $120 \mathrm{~mm}$ is the resonance length of the chassis at $900 \mathrm{MHz}$, thus optimum chassis-antenna working combination is achieved. However, increasing the chassis physical length is not a desirable option. From this perspective, we propose to control the ground plane length electrically using wires such that it resonates simultaneously with the antenna at the desired frequencies, thereby improving the overall antenna performance. The word "electrically" here means that the increase in the ground plane is done such that the handset device physical dimensions remain the same, while the RF performance is equivalent to that of a larger device.

We show next examples of different ways to achieve the goal of electrically extending the ground plane. The surface dimensions of the original ground plane, prior to applying our approach, are $100 \mathrm{~mm} \times 60 \mathrm{~mm}$. These dimensions are maintained in all examples. The closed wire/wires is/are electrically extended in the vertical direction, making use of the separation height between the ground plane and handset housing. This height is chosen to be within the height of a typical handset, $5 \mathrm{~mm}$. With this, the overall ground plane is now electrically larger than the original plane, even while keeping the handset exterior dimensions unchanged. The proposed models are presented in the following section, together with the antenna performances.

\section{Results and Discussion}

The proposed wire approach achieves two main objectives. The first is that the introduction and the careful design of these wires significantly improve the overall antenna performance. This is shown through the antenna bandwidth, the antenna efficiency, and SAR values. We also show that the wire extensions compare with a performance improvement when the ground plane is physically extended and this is shown through the near-field EM field intensity scans in the HAC plane. The second achieved objective is that the wire extensions do not affect the antenna characteristics itself but rather control the ground plane characteristics and hence its resonance. Therefore, the proposed approach does not introduce any interference and reradiation issues. This is shown through the far-field radiation patterns.

The proposed approach is shown in eight models. Model I is given in Figure 6(a) and models II-VIII are given in Figure 7. The performance improvements of these 


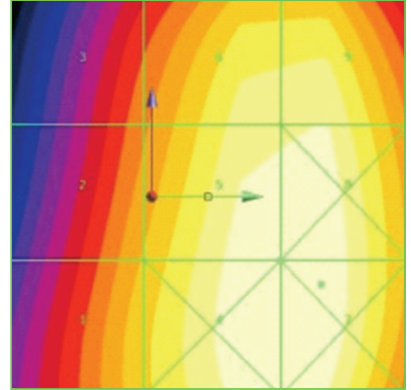

(a)

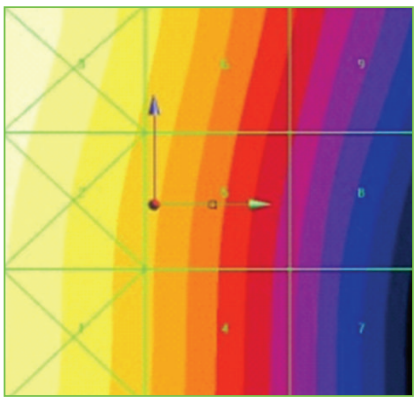

(b)



(c)

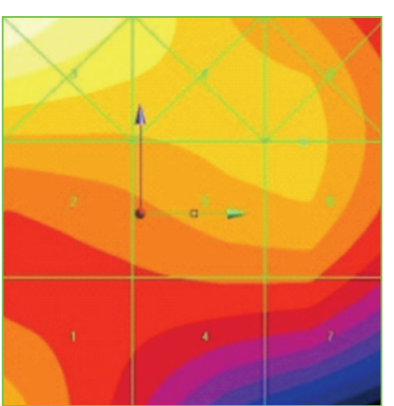

(d)
FIgURE 5: Electric and magnetic field scans of the AUT at chassis physical length of $120 \mathrm{~mm}$ : (a) HAC scan of $E$ field at $900 \mathrm{MHz}$, (b) HAC scan of $H$ field at $900 \mathrm{MHz}$, (c) HAC scan of $E$ field at $1880 \mathrm{MHz}$, and (d) HAC scan of $H$ field at $1880 \mathrm{MHz}$.

are listed in Tables 1 and 2 at frequencies $900 \mathrm{MHz}$ and $1880 \mathrm{MHz}$, respectively. A $6 \mathrm{~dB}$ bandwidth definition is used to measure the bandwidth improvement, and the SAR is computed based on the North American standard [20] through simulations [14].

Model I is the first model tested, and it presents an extension of the ground plane length. The presence of the wire loop at the end of the ground plane causes imbalance in the surface current, which then causes the current to extend further on the longitudinal edge, see Figures 6(b) and 6(c) compared to Figure 3. For this model we also compute the HAC scans based on [15] to examine the near-field properties as it become difficult to have a corresponding comparison using the surface currents only. These are compared to the HAC scan plane of the antenna with a physically extended ground plane of $120 \mathrm{~mm}$ instead of the original $100 \mathrm{~mm}$ length plane, see Figure 5. Notice that Figure 5 of the physically extended ground plane scans

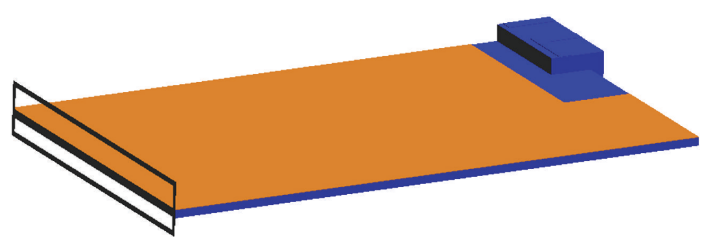

(a)

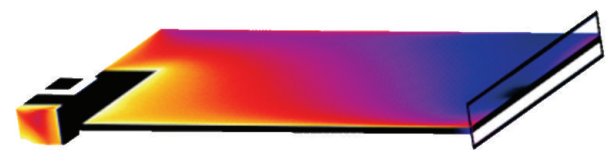

(b)

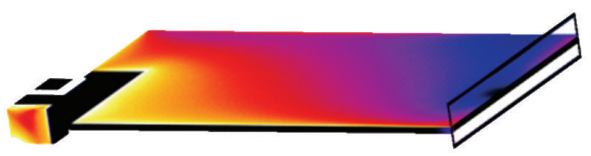

(c)

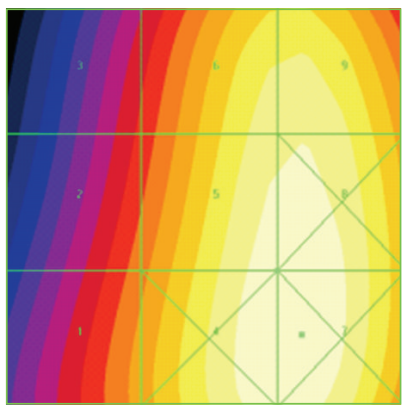

(d)

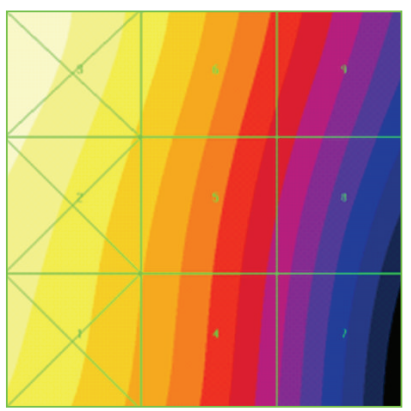

(e)

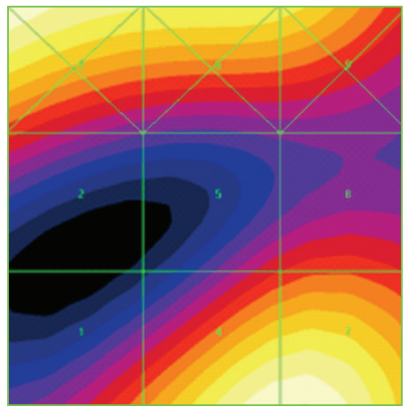

(f)

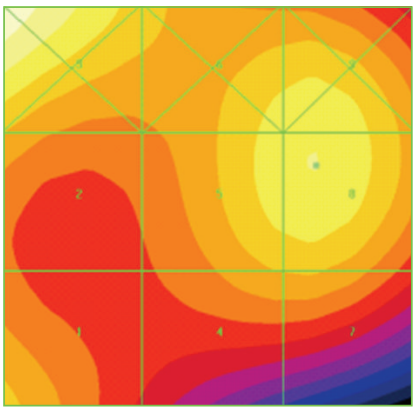

(g)

Figure 6: First configuration of the proposed approach: (a) model I, (b) current distribution at $900 \mathrm{MHz}$, (c) current distribution at $1880 \mathrm{MHz}$, (d) HAC scan of $E$ field at $900 \mathrm{MHz}$, (e) HAC scan of $H$ field at $900 \mathrm{MHz}$, (f) HAC scan of $E$ field at $1880 \mathrm{MHz}$, and (g) HAC scan of $H$ field at $1880 \mathrm{MHz}$. 


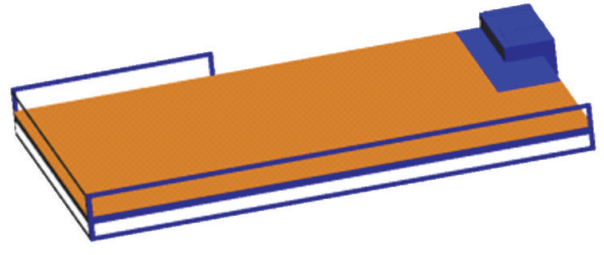

(a)

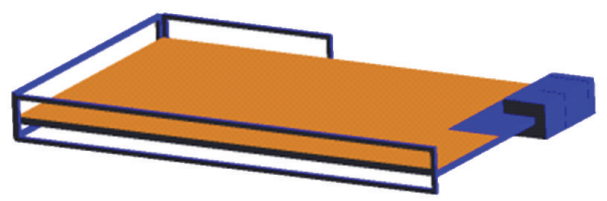

(c)

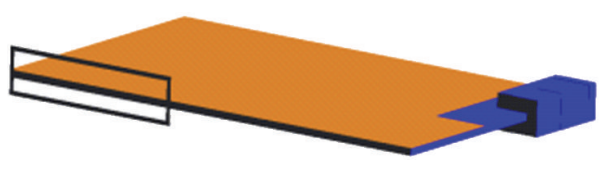

(e)

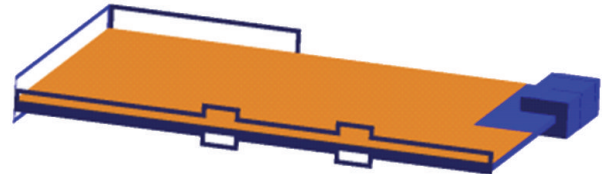

(b)

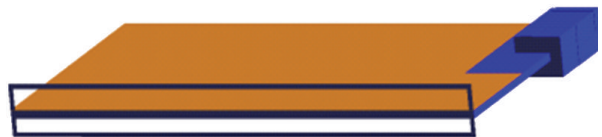

(d)

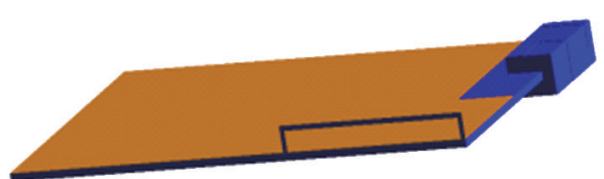

(f)

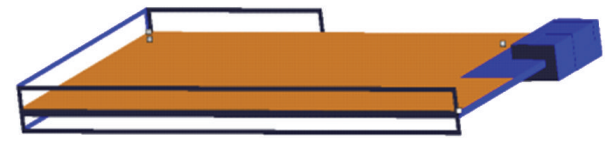

(g)

FIGURE 7: Configurations of the proposed approach: (a) model II, (b) model III, (c) model IV, (d) model V, (e) model VI, (f) model VII, and (g) model VIII.

TABLe 1: Percentage performance improvement at $900 \mathrm{MHz}$.

\begin{tabular}{lccc}
\hline Model & $\begin{array}{c}\text { Increase in } \\
\text { efficiency }\end{array}$ & $\begin{array}{c}\text { Increase in } \\
\text { bandwidth }\end{array}$ & $\begin{array}{c}\text { Reduction in } \\
\text { SAR }\end{array}$ \\
\hline I & 4.25 & 28.10 & 3.12 \\
II & 5.06 & 22.16 & 10.45 \\
III & 2.68 & 28.13 & 8.78 \\
IV & 2.63 & 14.81 & 11.99 \\
V & 7.70 & 56.44 & 8.11 \\
VI & 5.60 & 34.17 & 0.33 \\
VII & 4.15 & 44.77 & 5.50 \\
VIII & 6.15 & 35.23 & 9.87 \\
\hline
\end{tabular}

corresponds better with those of the electrically extended scans in Figures 6(d)-6(g) than to those of Figure 4 of the original antenna. Hence, we have achieved, with the wires, an effective extension comparable to that achieved with the addition of $20 \mathrm{~mm}$ to the ground plane length.

Different variations of the proposed wire approach are successfully tried and these are shown in Figure 7. In Figure 7(b), Model III, a bending of the wire, is used to form one of the sides of the loop. With this a reduction of the loop height is achieved for implementation in a slimmer handset. Figure 7(g), Model VIII, is similar to Model II shown in Figure 7 (a) but the difference is in the thickness of the wire used to form the closed loop. It is thicker in Model VIII. In both cases a good performance is achieved. Different sizes
TABle 2: Percentage performance improvement at $1880 \mathrm{MHz}$.

\begin{tabular}{lccc}
\hline Model & $\begin{array}{c}\text { Increase in } \\
\text { efficiency }\end{array}$ & $\begin{array}{c}\text { Increase in } \\
\text { bandwidth }\end{array}$ & $\begin{array}{c}\text { Reduction in } \\
\text { SAR }\end{array}$ \\
\hline I & 0 & -1.30 & 12.5 \\
II & -0.35 & 9.90 & 0 \\
III & 1.19 & -3.89 & 0.39 \\
IV & 0.96 & 5.24 & -1 \\
V & 1.25 & 11.66 & 1.47 \\
VI & 0.60 & 6.06 & 19.63 \\
VII & 0.67 & 5.10 & 18.73 \\
VIII & 0.77 & 8.10 & -0.3
\end{tabular}

and locations of the loop are tried in Figure 7(e), Model VI, and Figure 7(f), Model VII. These different implementations show the flexibility and ease of design of the proposed wire approach.

Comparisons of $2 \mathrm{D}$ radiation patterns for the AUT and all the proposed models are illustrated in Figure 8. These results show very similar $2 \mathrm{D}$ pattern, which suggests that the introduced wires do not cause interference and hence no reradiation occurs. Therefore, the far field radiation properties of the antenna are unaffected by the presence of the wires, and negative effects are avoided. The wires appear to predominantly affect only the ground plane chassis wavemodes.

Looking at the performance, Tables 1 and 2, the proposed approach offered an increase of up to $56 \%$ in bandwidth at 


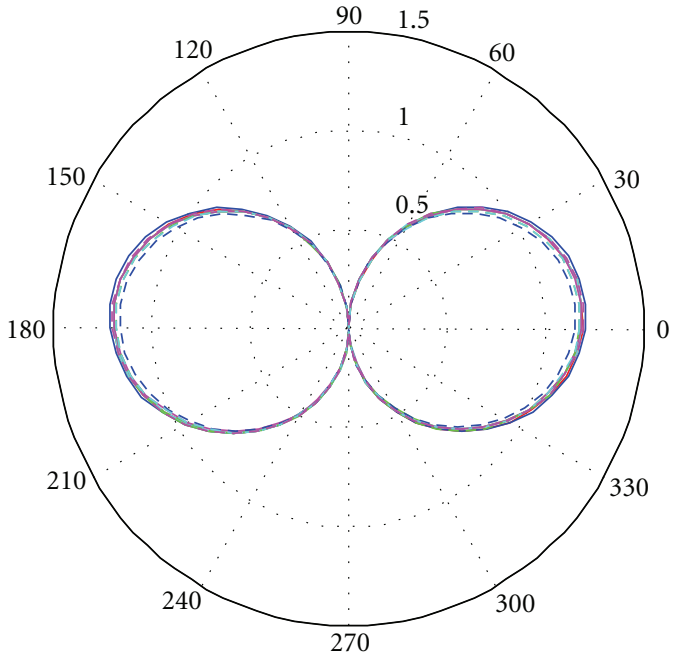

(a)

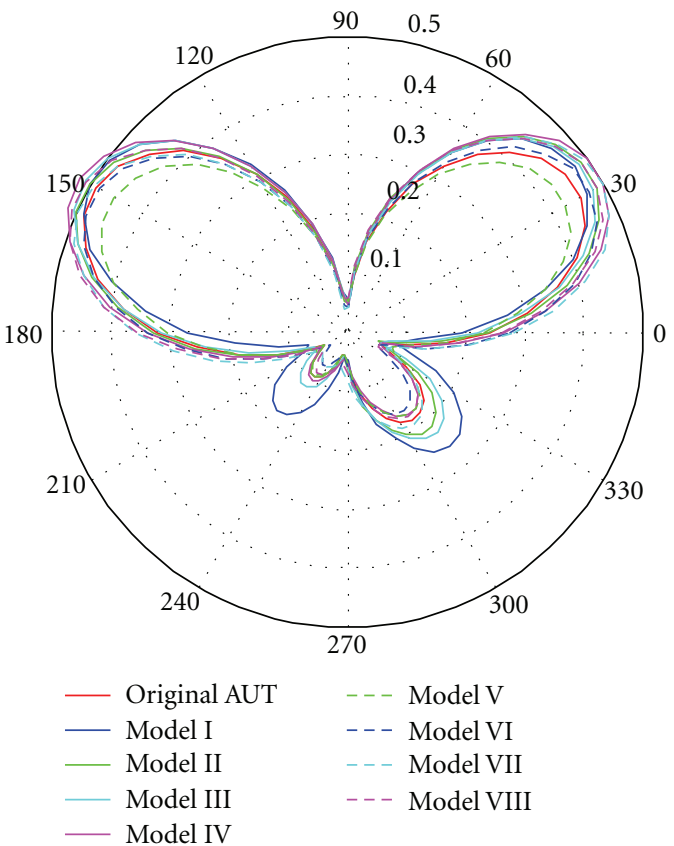

(c)

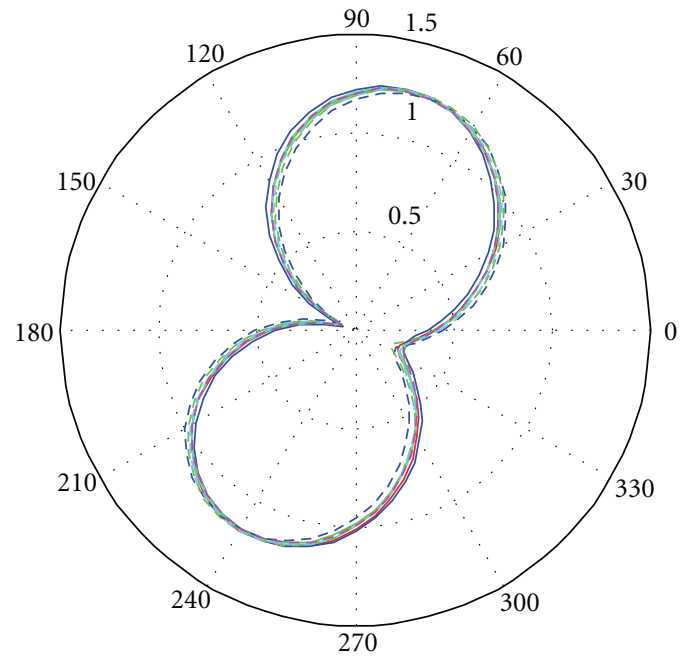

(b)

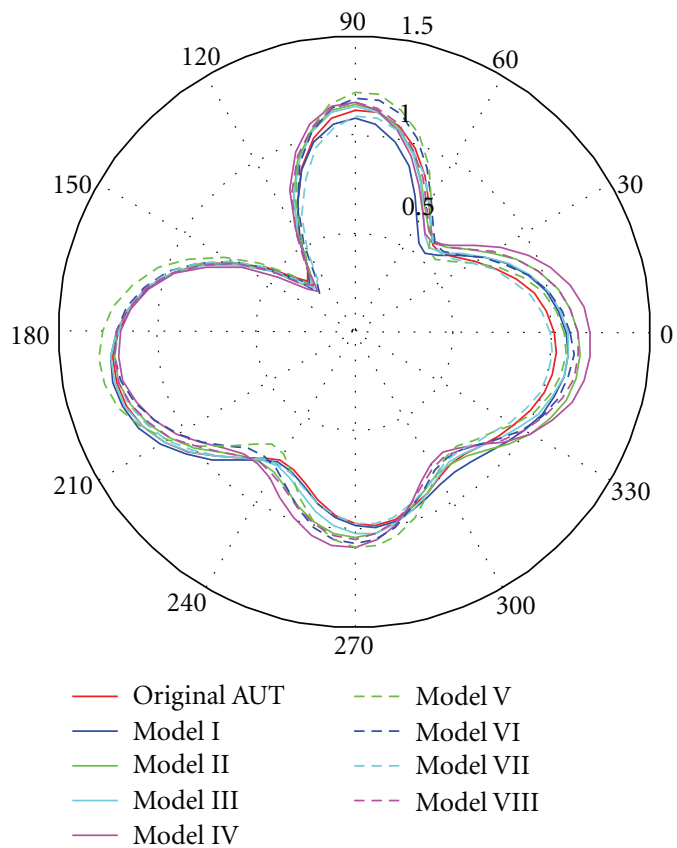

(d)

Figure 8: 2D radiation pattern comparison result: (a) 2D pattern in $\varphi$ plane at $900 \mathrm{MHz}$, (b) 2D pattern in $\theta$ plane at $900 \mathrm{MHz}$, (c) $2 \mathrm{D}$ pattern in $\varphi$ plane at $1880 \mathrm{MHz}$, (d) $2 \mathrm{D}$ pattern in $\theta$ plane at $1880 \mathrm{MHz}$, and (e) legend.

the $900 \mathrm{MHz}$ and a $12 \%$ increase at the higher $1880 \mathrm{MHz}$ frequency band. Currently, the proposed approach offers an improvement in the antenna efficiency without the need to enlarge the antenna structure; $8 \%$ efficiency was achieved at $900 \mathrm{MHz}$ and $1.3 \%$ was achieved at 1880 . This is significant, because the improvement in the lower frequency band was not achieved at the expense of performance at the higher frequency band or by degrading the bandwidth.

The implementation of our approach on a handset is easy and cost effective to manufacture. The wires constructing the virtual ground plane extension can be built into the inner surface of the handset device housing or can be designed as part of the device outer metal parts, for example, using part/all of the metal ring surrounding the perimeter of the device and connecting it through the device housing to the ground plane. Additionally, the improvements in the performance are achieved independent of the antenna structure, that is, no change is required in the design or in the location of the antenna. This permits the implementation of our approach without complicating the antenna design cycle.

\section{Conclusions}

We sidestep the traditional cost and limitations that are imposed when seeking a commonly desired benefit. The traditional cost for improving the antenna performance is an 
enlarged device, which may be undesirably large, thus limiting the implementation in practice. We accomplish the more desirable solution by electrically extending the length of the ground plane using wires that are placed outside the surface of the ground plane. This exploits a physical phenomenon that the proximity of the wires appears to create a single electrical surface with the ground plane, thereby achieving the enhanced radiation characteristics through controlling the ground plane chassis wavemodes to resonate with the antenna resonance. However, because the wires are outside the surface of the ground plane, they can be placed within the vertical volume normally enclosed by the device housing, without requiring the device housing to be lengthened. This avoids the traditional tradeoff. Performance improvements achieved are simultaneously seen on the antenna bandwidth, efficiency, and the lower specific absorption rates. The proposed wire approach is successfully illustrated in eight models and at a low- and a high-frequency bands.

\section{Acknowledgment}

The authors would like to thank Dr. Houssam Kanj from Advanced Technology, Research In Motion Limited, for his comments and fruitful discussions.

\section{References}

[1] O. Kivekäs, J. Ollikainen, T. Lehtiniemi, and P. Vainikainen, "Bandwidth, SAR, and efficiency of internal mobile phone antennas," IEEE Transactions on Electromagnetic Compatibility, vol. 46, no. 1, pp. 71-86, 2004.

[2] T. Taga and K. Tsunekawa, "Performance analysis of a built-in planar inverted $\mathrm{F}$ antenna for $800 \mathrm{MHz}$ band portable radio units," IEEE Journal on Selected Areas in Communications, vol. 5, no. 5, pp. 921-929, 1987.

[3] K. Sato, K. Matsumoto, K. Fujimoto, and K. Hirasawa, "Characteristics of a planar inverted-F antenna on a rectangular conducting body," Electronics and Communications in Japan, Part I, vol. 72, no. 10, pp. 43-51, 1989.

[4] T. Taga, "Analysis of planar inverted-F antennas and antenna design for portable radio equipment," in Analysis Design, and Measurement of Small and Low Profile Antennas, K. Hirasava and M. Heneishi, Eds., Artech House, 1992.

[5] P. Vainikainen, J. Ollikainen, O. Kivekäs, and I. Kelander, "Resonator-based analysis of the combination of mobile handset antenna and chassis," IEEE Transactions on Antennas and Propagation, vol. 50, no. 10, pp. 1433-1444, 2002.

[6] D. Manteuffel, A. Bahr, and I. Wolff, "Investigation on integrated antennas for GSM mobile phones," in Proceedings of the Millennium Conference on Antennas Propagation, 2000.

[7] D. Manteuffel, A. Bahr, D. Heberling, and I. Wolff, "Design considerations for integrated mobile phone antennas," in Proceedings of the International Conference on Antennas Propagation, pp. 252-256, 2001.

[8] A. T. Arkko and E. A. Lehtola, "Simulated impedance bandwidths, gains, radiation patterns and SAR values of a helical and a PIFA antenna on top of different ground planes," in Proceedings of the International Conference on Antennas Propagation, pp. 651-654, 2001.
[9] S. M. Ali and H. Gu, "Chassis wavemode effects on hearing aid compatibility at $900 \mathrm{MHz}$," in Proceedings of the International Conference on Antennas Propagation, pp. 651-654, July 2010.

[10] S. M. Ali and H. Gu, "Chassis wavemode effects on hearing aid compatibility (HAC) in the handset".

[11] R. Hossa, A. Byndas, and M. E. Bialkowski, "Improvement of compact terminal antenna performance by incorporating open-end slots in ground plane," IEEE Microwave and Wireless Components Letters, vol. 14, no. 6, pp. 283-285, 2004.

[12] T. W. Chiou and K. L. Wong, "Designs of compact microstrip antennas with a slotted ground plane," in IEEE Antennas and Propagation Symposium, pp. 732-735, July 2001.

[13] S. M. Ali and H. Kanj, "Hex-band antenna for slim handheld device applications," Microwave and Optical Technology Letters, vol. 51, no. 11, pp. 2527-2530, 2009.

[14] SEMCAD-X Reference Manual, Zeughausstrasse 43, Schmid \& Partner Engineering AG (SPEAG), Zurich, Switzerland, 2007.

[15] ANSI C63.19-2007 (Revision of ANSI C63.19-2006), American national standard methods of measurement of compatibility between wireless communications devices and hearing aids, June 2007.

[16] T. Taga and K. Tsunekawa, "Performance analysis of a built-in inverted-F antenna for $800 \mathrm{MHz}$ band portable radio units," IEEE Journal on Selected Areas in Communications, vol. 5, no. 5, pp. 921-929, 1987.

[17] H. Nakano, N. Ikeda, Y. Y. Wu, R. Suzuki, H. Mimaki, and J. Yamauchi, "Realization of dual-frequency and wideband VSWR performances using normal-mode helical and inverted-F antennas," IEEE Transactions on Antennas and Propagation, vol. 46, no. 6, pp. 788-793, 1998.

[18] T. Tag, Analysis, Design, and Measurement of Small and LowProfile Antennas, Artech-House, Boston, Mass, USA, 1992.

[19] W. Geyi, Q. Rao, S. Ali, and D. Wang, "Handset antenna design: practice and theory," Progress in Electromagnetics Research, vol. 80, pp. 123-160, 2008.

[20] IEEE Std. 1528-2003, Recommended Practice for Determining the Peak Spatial-Average Specific Absorption Rate (SAR) in the Human Head from Wireless Communications DevicesMeasurement Techniques, Institute of Electrical and Electronics Engineers, New York, NY, USA, 2003. 

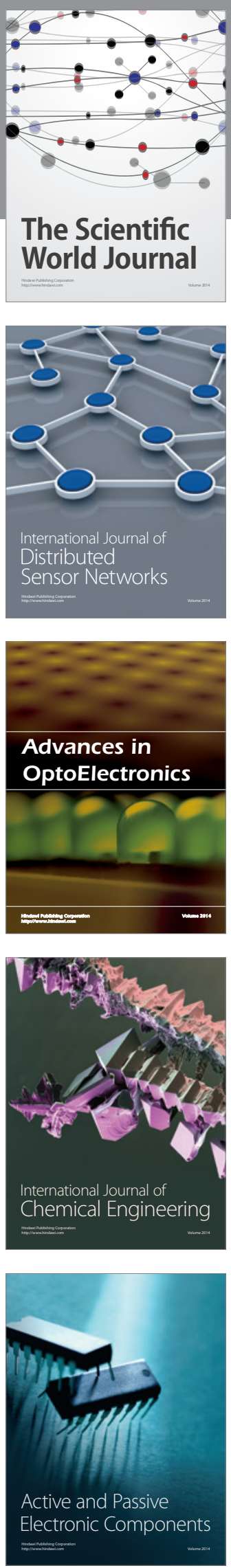
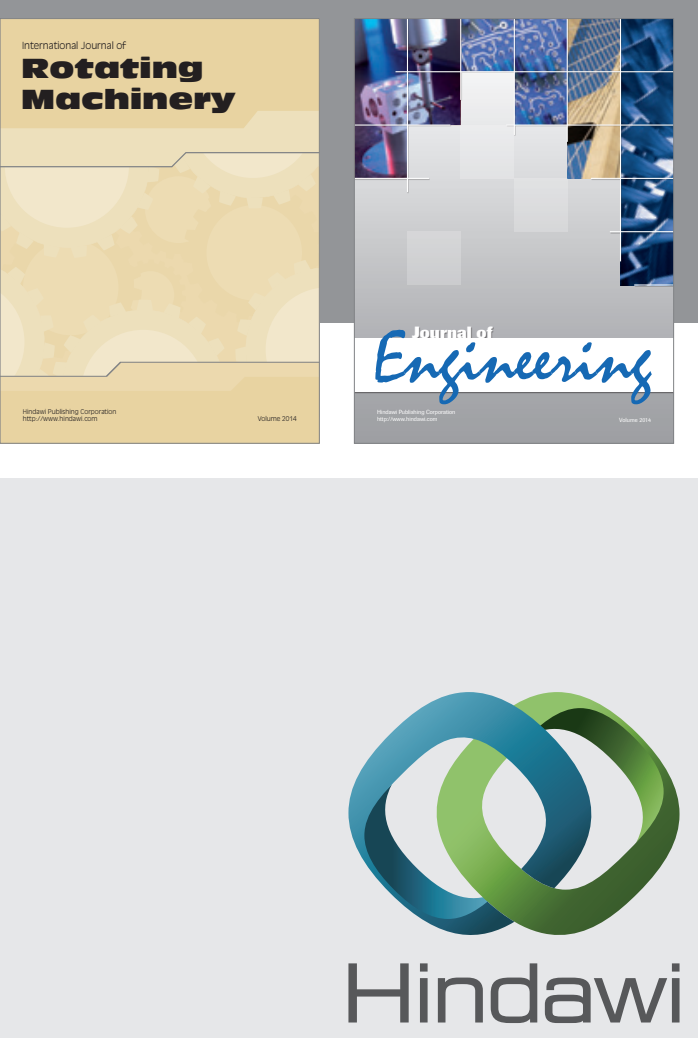

Submit your manuscripts at

http://www.hindawi.com
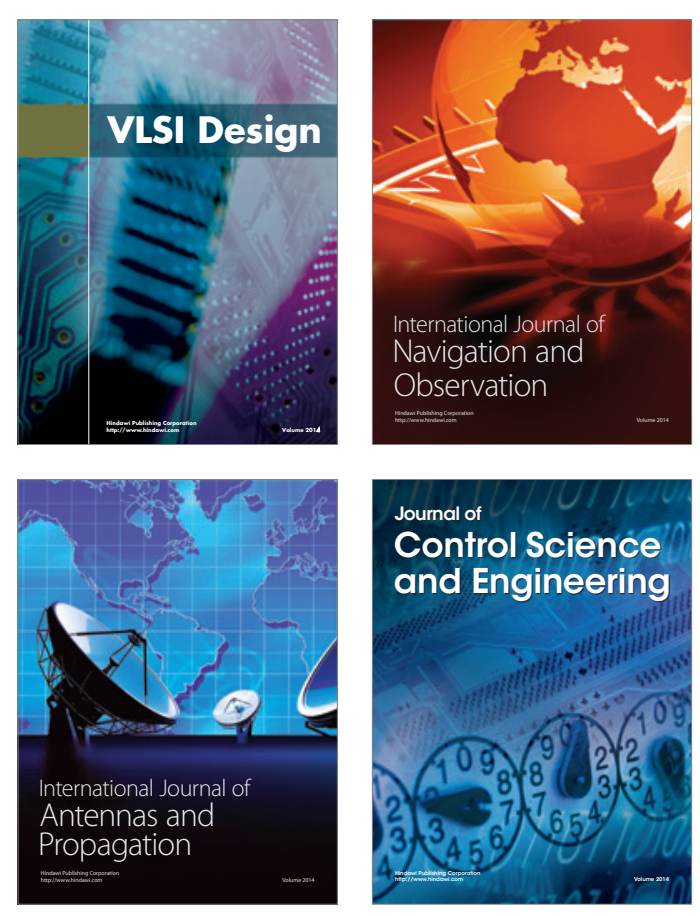
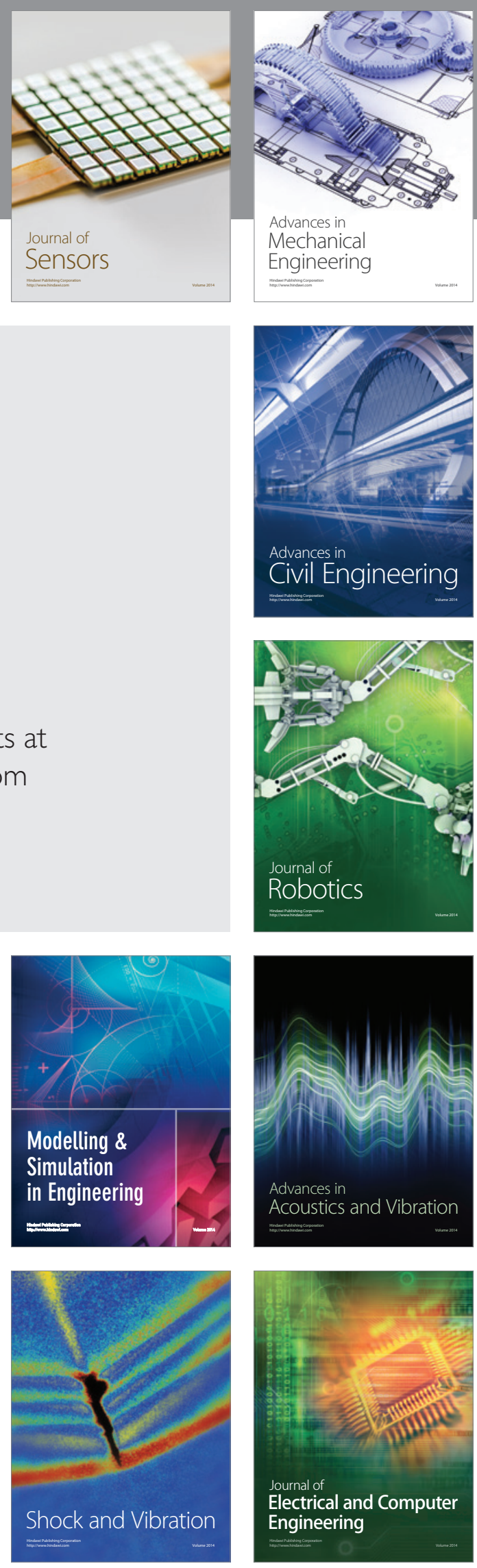